\title{
Rapid Prototyping of Planning, Learning and Control in Physical Human-Robot Interaction
}

\author{
Martin Lawitzky, José Ramón Medina Hernández, and Sandra Hirche
}

\section{Introduction}

Recent advances in robotics research render the prospect of robotic assistants entering weakly structured, daily-life domestic, and industrial scenarios within reach of the next decade. Some of the most challenging problems are in the area of proactive and goal-directed physical robotic assistance to the human. The physical coupling between the robot and the human imposes particular challenges in terms of real-time capability, adaptability, uncertainty of human behavior, and safety of the decision making and negotiation algorithms.

It is well known that the estimation of the human partner's intention is crucial for intuitive physical human-robot interaction [7]. Methods to estimate human intention based on the minimum-jerk assumption [8] are often deployed [19,4]. Within the programming-by-demonstration framework a significant body of methods to learn motion patterns from human demonstration, to recognize such and to estimate human intention are developed. The closest related works are the Locally Weighted Regression [26], Dynamic Movement Primitives [13, 23], Locally Weighted Projection Regression [31], Gaussian Processes [32], and Gaussian Mixture Models [10]. The Gaussian Mixture Regression approach is recently applied to a coaching setting in physical human-robot interaction [18], see [2] for an excellent survey. Motion planning in the context of physical robotic assistants finds only little reference in the literature. Taïx et al. recently presented a method improve the sampling strategy of a path planner through haptic input while supporting the human guide to find the goal in a two-dimensional virtual reality scenario [29]. Feedback motion planning for haptic guidance using a cell-decomposition method is proposed by [24] in the context of computer-aided design. So far, most of the existing works investigate individual algorithms for planning [29, 24], learning/prediction [19, 4, 10, 18], and

Martin Lawitzky, José Ramón Medina Hernández, and Sandra Hirche Technische Universität München, Inst. of Automatic Control Eng., 80290 Munich, Germany, e-mail: $\{$ ml, medina, hirche $\} @$ tum.de 
control $[27,16,15,15,34,6]$ for physical human-robot interaction (pHRI) in a separate way. Only very few works consider the combination of learning and control in pHRI $[11,20]$. Another substantial difference in pHRI to classical robotics is the need for human user studies to evaluate the robot behavior in a human-centered way.

The contribution of this work is twofold: i) We present a rapid prototyping experimental environment for quick and easy development of integrated planning, learning and control algorithms for pHRI, their objective evaluation, and psychological evaluation in large-scale user studies. It includes a high-fidelity two degrees-of-freedom device and seamless transfer to a highly integrated bimanual mobile robot with a modular software system, which is used in studies with naïve users. ii) We present novel results on the combination of planning and learning mechanisms in physical human-robot interaction using this rapid-prototyping setup. In particular, the complementary properties of a learning and motion prediction algorithm [3] and a feedback-planning algorithm [33] are examined, in order to derive strategies for a synergetic combination of these two different approaches.

Most of the existing approaches toward motion generation for robotic physical assistants can be described as either a) planning-based approaches or b) approaches based on the reproduction of human behavior from observations. Feedback-planning approaches suit the need for instant decision making, incremental learning algorithms allows to adapt to a human partner's (changing) preferences. As prototypical task we investigate the cooperative transport of an object from an initial to a final configuration through a cluttered environment. Similar settings are found in mobility assistance to humans, physical rehabilitation, and computer-aided assembly. Our proposed approach is structured as five-layered framework embedding pluggable algorithms for the individual layers. The algorithms are evaluated in human user studies on a two-dimensional setup as well as in a full-scale six-dimensional task with a human cooperating with a mobile manipulator.

The remainder of this paper is organized as follows: After the problem setting and our approach are described in Section 2, the results are presented in Section 3. Section 4 explains the experimental setup used, followed by the main insights of this work in Section 5.

Notation: Bold characters are used for vectors and matrices. The configuration space of the manipulated rigid object is denoted $\mathscr{C}$ which is a manifold $\mathscr{C}=\mathbb{R}^{2}$ in two-dimensional case without rotation and $\mathscr{C}=\mathbb{R}^{3} \times \mathbb{R P}^{3}$ in the three-dimensional case with rotation. The obstacle region is denoted $\mathscr{C}_{\text {obs }} \subseteq \mathscr{C}$. The leftover configurations are called free space which is denoted $\mathscr{C}_{\text {free }}=\mathscr{C} \backslash \mathscr{C}_{\text {obs }}$.

\section{Technical Approach}

The quality of assistance benefits from goal-oriented robot behavior - in contrast to purely reactive behavior - as shown for example in a cooperative load transport task [17, 22]. For simplicity and clarity we focus here on the physical assistance in 
the redundant degrees of freedom of the task, i.e. a more pro-active robot reduces the required human effort to solve the task. Our proof-of-concept studies show that planbased [14] as well as motion reproduction strategies [20,21] can successfully generate goal-oriented behaviors in full-scale cooperative transport tasks and improve assistance. These results expose complementary properties such as adaptation to human preferences in case of learning approaches and explicit consideration of environment knowledge in planning-based approaches indicating the potential for synergies. In this work we experimentally investigate representative algorithms from each field, the sampling-based neighborhood graph (SNG) feedback planning [33] and Gaussian mixture regression (GMR) [3] uncovering their complementary strenghts in the context of physical robotic assistance in cooperative manipulation tasks. In addition, we will present a novel approach combining those two approaches and evaluate this experimentally in a user study using the rapid prototyping setup.

Problem setting We consider the problem of a human-robot dyad cooperatively moving an object from a start configuration $\mathbf{x}(0) \in \mathscr{C}_{\text {free }}$ to a different final configuration $\mathbf{x}\left(t_{\text {goal }}\right) \in \mathscr{C}_{\text {free }}$ through a cluttered environment.

The articulated geometric model with six DoF approximating the human, object and robot for a cooperative transport task is depicted in Fig. 1. The object itself can be moved in all six degrees of freedom (DoF). For simplicity in this work we assume that a human partner and a robot control their posture such that they keep a constant configuration relative to the table on the two-dimensional ground plane $\left(x_{0}, y_{0}\right)$. Aspects of approximation of person-object-robot representations for motion planning are discussed in [5].

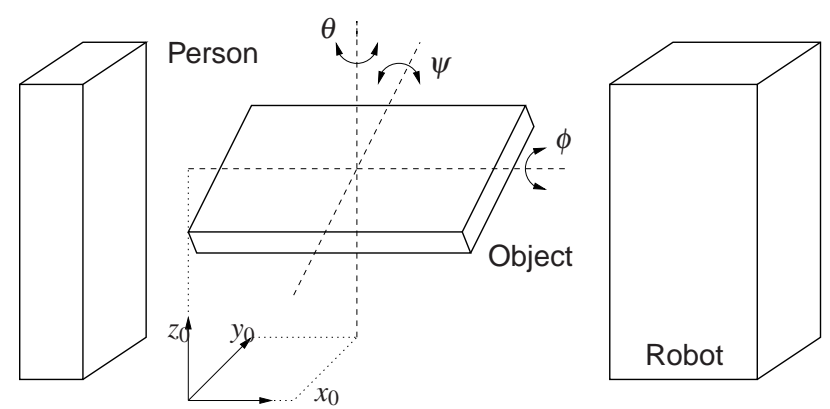

Fig. 1: 6-DoF reduced geometric model of the cooperation partners and the object used for calculation of free configuration space $\mathscr{C}_{\text {free }}$

We assume a robotic assistant is feedback-controlled such that the commonly manipulated object follows a virtual object impedance that is rendered with inertia matrix $\mathbf{M}$, and virtual viscous friction $\mathbf{D}$.

$$
\mathbf{M} \ddot{\mathbf{x}}+\mathbf{D} \dot{\mathbf{x}}=\mathbf{u}_{h}+\mathbf{u}_{r},
$$


where $\mathbf{x}$ denotes the object pose and $\mathbf{u}_{h}$ and $\mathbf{u}_{r}$ the effective wrenches by human and robot, respectively. Both cooperation partners are assumed to have their individual plans $\tau_{\mathbf{x}(t), h}(s)$ and $\tau_{\mathbf{x}(t), r}(s)$ with index $s \in[0,1]$. Due to continuous plan negotiation the executed path regularly deviates from the initially computed plan $\tau_{\mathbf{x}(0), h}(s)$ and $\tau_{\mathbf{x}(0), r}(s)$ and the plan has to be re-computed at $x(0)$, which is indicated by the subscript.

To address the plan-generation problem, we investigate a learning-based and a planning-based strategy. In a learning-based approach the robot initially behaves passive, i.e. $\mathbf{u}_{r}=0$ during the task execution and observes the executed trajectory which can be assumed to be close to the human partner's intended path $\tau_{\mathbf{x}(0), h}(s)$. In subsequent trials the robot adopts this as its own motion plan $\tau_{\mathbf{x}(0), r}(s) \leftarrow \tau_{\mathbf{x}(0), h}(s)$. Note that the space of learned trajectories is generally only a subspace of the free space, but generally the subspace of learned trajectories is larger than the space of demonstrated trajectories $\mathscr{C}_{\text {demo }} \subseteq \mathscr{C}_{\text {learn }} \subseteq \mathscr{C}_{\text {free }}$, see Fig. 2. Algorithms for motion learning and reproduction have limited generalization capabilities and can thus cover regions neighboring the demonstrated trajectories.

In a planning-based approach the robot computes its motion plan based on environmental constraints and possibly under consideration of manipulability constraints by the human partner. In consequence the robot plan will generally differ from the human plan $\tau_{\mathbf{x}(t), r}(s) \neq \tau_{\mathbf{x}(t), h}(s)$ and an online negotiation is required. In

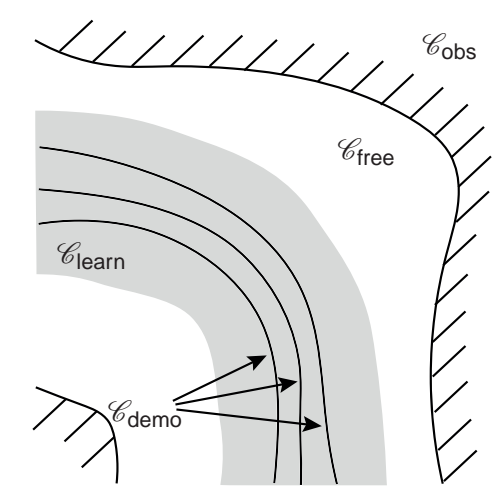

Fig. 2: Subsets of the configuration space $\mathscr{C}=\mathbb{R}^{2}$

contrast to path planning, feedback motion planning strategies prepare the goaldirected robotic assistant for this property through a continuous adjustment of the robot's desired path $\tau_{\mathbf{x}(t), r}(s)$ from the current configuration to the goal configuration.

A rapid prototyping experimental system is developed in which complex algorithms for pHRI can be tested plug-and-play - also in user studies - using modular building blocks. In a first stage those algorithms are tested on a 2-Dof haptic device in a virtual haptic environment and later directly transferred to a large-scale an- 
thropomorphic robotic system with integrated visual tracking and laser-range based localization schemes, see Section 4. Interchangeable control blocks such as an admittance and a novel risk-sensitive optimal-feedback controller [21], are used.

We propose a five-layered taxonomy for the modules of physical robotic assistants. The topmost abstraction layer observes the environment and the interaction partner and generates models of both. States of the models are estimated and models are updated within this layer. The second layer generates desired motion patterns given a common goal for the cooperative transport and the interaction state. Multiple implementations of this layer can be evaluated concurrently. The resulting directions of motions are merged in the deliberative layer that decides among different generated motion patterns. From the robot's desired trajectory, the resulting necessary external forces on the commonly manipulated object are calculated. The required effort is distributed among the cooperating partners. The lowest abstraction layer handles the reactive control of the human-robot dyad. It ensures that any deviation between the human intention and the robot's desired motion results in smooth force/motion trajectories.

Table 1: Layers for rapid prototyping of physical robotic assistants

\begin{tabular}{ll}
\hline Layer & Example implementations \\
\hline Perception and modeling layer & Plan negotiation, intention estimation \\
Generative layer & Feedback planning, motion reproduction \\
Deliberative layer & Blending, multi-criterion decision making \\
Interaction control layer & Effort sharing, redundant object-pose control \\
Reactive layer & Admittance control, position-based force control \\
\hline
\end{tabular}

With our rapid prototyping experimental system, different alternative implementations of each layer are experimentally evaluated: Joint planning through automatic segmentation and verbal communication in combination with intention recognition implements perception and modeling of the task and the partner behavior [20]. Motion generated from the learned human motion model and the estimated human intention or plan-based alternatives [14] implement the generative layer. Two alternatives are further investigated in this work. Concurrent implementations of the generative layer generate different desired motion trajectories. The decision on either one single option or a blend of multiple options is left to the deliberative layer. Potential methods for decision and blending are proposed in this work. The interaction control layer can be implemented as static role allocation (leader/follower) [17] or dynamic effort sharing strategy [22]. Alternatively, a risk-sensitive optimal feedback control scheme can be applied to generate an intuitive robot force contribution depending on observed human execution variability [21]. In the following, two concurrent implementations of the generative layer, the sampling-based neighborhood graph (SNG) for feedback planning [33] and the time-based HMM (tHMM) learn- 
ing, recognition and a learning-based framework $[18,20]$ are briefly reviewed, and two alternatives for the deliberative layer are proposed.

Feedback motion planning A feedback motion planning algorithm generates a feedback function $\mathbf{K}(\mathbf{x})$ for all positions $\mathbf{x} \in \mathscr{C}_{\text {free }}$ in the accessible configuration space $\mathscr{C}_{\text {free }}$. The SNG is a very comprehensible method, sufficiently efficient to cover higher-dimensional configuration spaces applicable for settings with 6dimensional object poses at a viable resolution as required for our large-scale scenario. In brief, the method consists of the following steps:

For each new map given by $\mathscr{C}_{\text {free }}$

1. Cover $\mathscr{C}_{\text {free }}$ with $n$-dimensional hyper balls until a specified percentage $a$ (e.g. 99\%) is covered with desired probability $P_{c}$ (e.g. $99 \%$ ).

2. Create a graph representing connectedness of hyper balls.

For each new goal $\mathbf{x}_{\text {goal }}$.

3. Identify hyper ball containing $\mathbf{x}_{\text {goal }}$

4. Generate priority graph e.g. using Dijkstra's algorithm

During execution

5. Calculate direction to next best hyper ball

Given a desired magnitude of velocity and a desired maximum robot force level, the force ${ }^{1}$ vector $\mathbf{u}_{\text {plan }}$ is calculated straightforwardly with the virtual object's inverse dynamical model.

Learning from demonstration Learning-based approaches to active physical assistance aim to imitate the human partner's behavior during demonstrations in order to take over the task effort instead of planning a task-directed behavior. In contrast to planning-based approaches, this straightforwardly adapts to human preferences.

The tHMM framework is shown to encode human trajectory demonstrations efficiently and most importantly constrains motion reproduction to the configuration space $\mathscr{C}_{\text {learn }} \subseteq \mathscr{C}_{\text {free }}$ (i.e. a zone of influence) around the demonstrated configurations $\mathscr{C}_{\text {demo }} \subseteq \mathscr{C}_{\text {learn }}$. As proposed in [18], we combine Hidden-MarkovModels (HMM) with Gaussian Mixture Regression (GMR) in an incrementallearning framework that allows modeling and reproduction of smooth trajectories and is well suited to ensure intuitive motion generation in physical human-robot cooperation [20].

We investigate, how the complementary properties of these two algorithms for motion generation discussed above can be beneficially combined such that synergies arise.

Two methods to exploit synergies are derived in the following:

\footnotetext{
${ }^{1}$ Force in the $2 \mathrm{D}$ case, wrench in the $6 \mathrm{D}$ case
} 
Prediction-quality-based homotopy blending exploits the probabilistic characteristics of the motion recognition and prediction algorithm. As recognition uncertainty grows, the goal-oriented fall-back solution generated by the feedback planning algorithm is activated. A homotopy $\mathscr{H}(t, \gamma)$ blends between the two output functions:

$$
\mathbf{u}_{\mathrm{r}}=\gamma \mathbf{u}_{1}+(1-\gamma) \mathbf{u}_{\mathrm{p}}
$$

with prediction certainty $\gamma \in[0,1]$. The outputs of the planning algorithm and the learning algorithm are denoted $\mathbf{u}_{\mathrm{p}}$ and $\mathbf{u}_{1}$ respectively. Similar to [30], we regard the the unnormalized likelihood of the state estimate as a measure for certainty. This strategy overcomes the limitation of the learning-based algorithm to produce active task contributions only within the zone of influence $\mathscr{C}_{\text {learn }}$ and blends continuously into the feedback motion plan valid for the entire accessible configuration space $\mathscr{C}_{\text {free }}$.

Cost-based fusion of strategies is based on the parallel evaluation of the strategies and a cost-based fusion using a sophisticated hierarchical multi-criteria decision making (MCDM) algorithm on the efficient (Pareto) frontier. Two different utility criteria are evaluated, one from the planning-based approach and one from the learning-based approach. The utility function $\mathscr{U}(\mathbf{x}, \dot{\mathbf{x}}, \mathbf{u})$ of the planning algorithm is calculated through simulation of the effects of different forces $\mathbf{u}$ on the cost to go by querying the feedback plan. The utility is maximized for force vectors along the planning algorithm output $\mathbf{u}_{\mathrm{p}}$ and and minimized for the opposite direction. The utility value of 0 is cost-neutral and is reached for directions orthogonal to the planned direction of motion.

The utility function of the learning-based algorithm is described by the prediction certainty $\gamma \in[0,1]$. The certainty value of 1 is reached at the mean direction of the motion prediction according to the unnormalized likelihood of the prediction.

A hierarchical MCDM-scheme inspired by [25] is deployed: The output of the learning-based approach is evaluated for its utility $\mathscr{U}\left(\mathbf{x}, \dot{\mathbf{x}}, \mathbf{u}_{l}\right)$. In case the utility is positive, the output of the learning-based approach is accepted, $\mathbf{u}_{r}=\mathbf{u}_{l}$. Otherwise, the closest direction to $\mathbf{u}_{l}$ on the efficient frontier with non-negative utility $\mathscr{U}\left(\mathbf{x}, \dot{\mathbf{x}}, \mathbf{u}_{r}\right) \geq 0$ is selected

$$
\mathbf{u}_{r}=\underset{\mathbf{u} \in\left\{\mathbf{u}_{i} \mid \mathscr{\mathscr { U }}\left(\mathbf{x}, \dot{\mathbf{x}}, \mathbf{u}_{i}\right) \geq 0\right\}}{\arg \max } \mathbf{u}^{T} \mathbf{u}_{l} .
$$

Control architecture The control algorithm embedding the algorithms proposed above is depicted in Fig. 3. Both methods continuously produce wrenches $\mathbf{u}_{p}, \mathbf{u}_{l}$ that act on a virtual admittance in superposition with the human partner's force $\mathbf{u}_{h}$ exerted through the object on the end effector of the robot. 


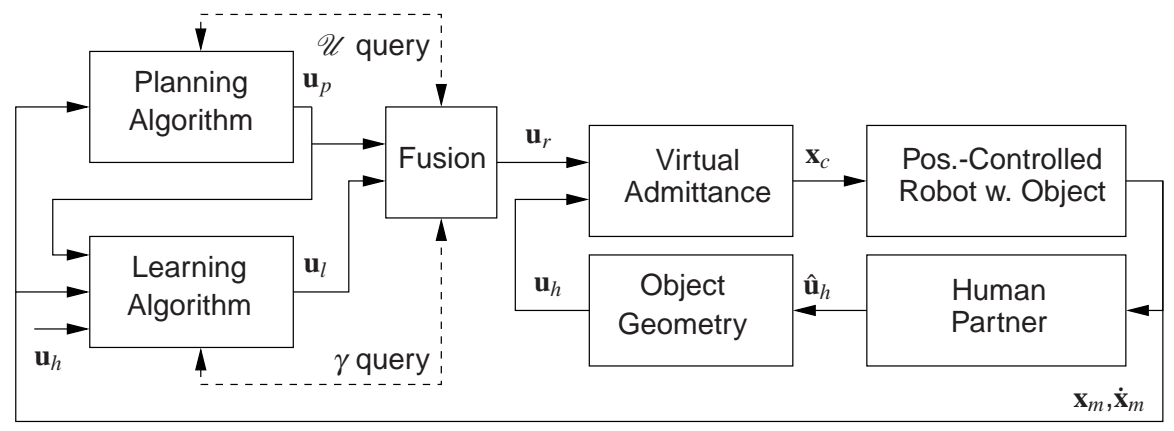

Fig. 3: Architecture for integrated planning, learning and control in pHRI

Fig. 4: Evolution of quantitative parameters over trials

(a) RMS path deviation

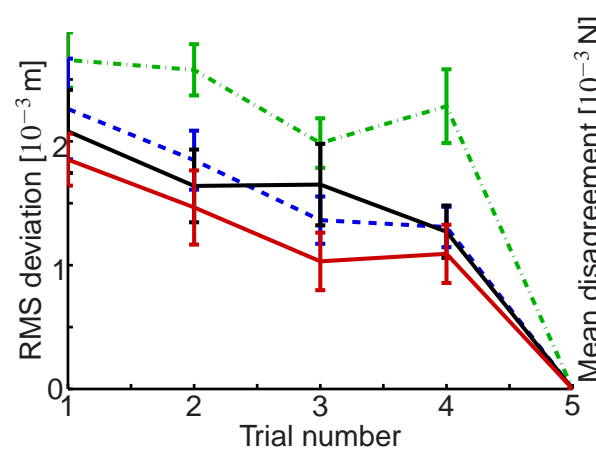

(b) Disagreement

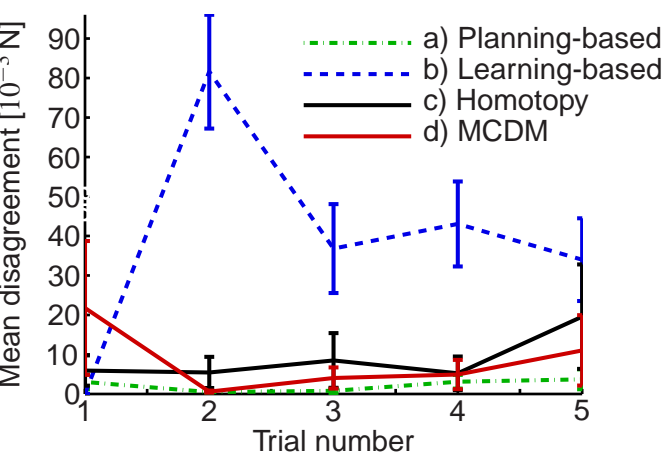

(c) Completion time

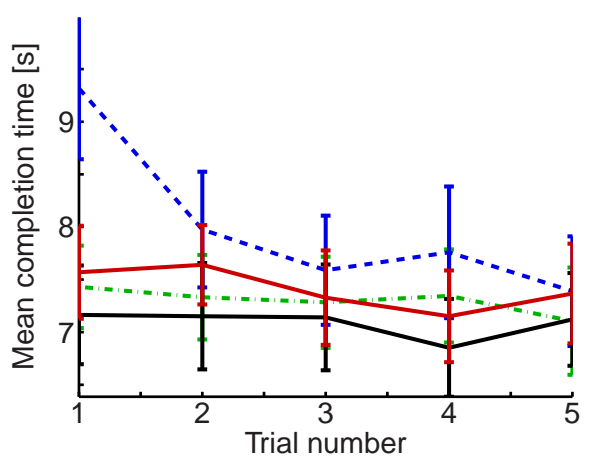

\section{Results}

The proposed approaches are evaluated in experiments with cooperating humans. This section describes the evaluation criteria and the quantitative results. For illus- 
tration, the feedback plan as well as the learned task models in pHRI in the small scale experiment in 2DoF are shown in Figs. 5(a)-5(c), and for the large scale setup in 6DoF in Figs. 5(d) -5(f).

A small pilot study in a virtual reality scenario was conducted yielding the results depicted in Fig. 4. Seven non-paid participants (age mean: 27.1, std: 1.5) were asked to move a virtual point mass object of $100 \mathrm{~kg}$, through a simple maze from a starting configuration to a final configuration through the scene without colliding with the virtual obstacles visually and haptically displayed. The virtual object was exposed to a virtual viscous friction of $400 \mathrm{Ns} / \mathrm{m}$.

Each participant repeated the experiment five times per each of five conditions after five training trials without assistance: a) assisted by feedback planning, b) assisted by learning-based algorithm, c) prediction-quality-based homotopy blending, d) multi-criteria decision making, and e) learning-based, but pre-initialized with simulated feedback planning.

Quantitative measures We evaluate the following criteria in order to rate the performance of the proposed approaches:

- Mean root-mean-square (RMS) deviation from the participant's path $\tau_{\mathbf{x}, H 5}$ after five trials

$$
x_{\mathrm{RMS}}=\sqrt{\frac{1}{T} \int_{0}^{T}\left\|\mathbf{x}(t)-\underset{\mathbf{x}_{\tau} \in \tau_{\mathbf{x}, H 5}}{\arg \min }\right\| \mathbf{x}(t)-\mathbf{x}_{\tau}\|\|^{2}} d t .
$$

This measure represents the required amount of adaptation by the human from trial to trial. Larger values indicate a higher amount of adaptation from trial to trial.

- Mean disagreement $u_{D}$ which can be defined orientation invariant:

$$
\mathbf{u}_{D}= \begin{cases}\frac{-\left(\mathbf{u}_{h}\right)}{\left\|\mathbf{u}_{h}\right\|} \cdot \mathbf{u}_{r}, & \text { if } \mathbf{u}_{h} \cdot \mathbf{u}_{r}<0 \wedge \mathbf{u}_{h} \neq \mathbf{0} \\ 0, & \text { otherwise. }\end{cases}
$$

Larger values of disagreement $u_{D}$ indicate that the human and the robotic partner produce a higher amount of counteracting, and therefore inefficient forces.

- Mean completion time $T_{\text {mean }}$ as indicator of efficiency of the cooperation.

The evaluation of the properties of the planning-based and learning-based approaches in terms of physical measures are depicted in Fig. 4. Regarding the RMS deviation over trials, it is visible that the conditions c) Homotopy blending and d) MCDM require the least adaptation and are in a similar range as the b), the pure learning-based approach. Condition a), the planning-based approach leads to stronger adaptation of the human. The measurements of mean disagreement show that the conditions a), c) and d) perform equally well. The mean disagreement of these conditions is lower than in the pure learning-based condition. The completion times of the fusion strategies c) and d) are similar to those of the planning-based approach and lower than in the learning-based strategy. 
Discussion: From the quantitative results it is visible that the fusion strategies c) and d) combine the strengths of the planning-based and the learning-based approaches a) and b). These fusion methods outperform the planning-based approach regarding the required adaptation of the human and leads to small disagreement compared to the learning-based approach. The completion time under these conditions is on the level of the planning approach throughout all trials and is significantly lower than the unassisted condition in the first trial of the learning-based approach.

\section{Experimental Setups}

The approaches are first tested in a small-scale 2DoF virtual scenario and then transferred to a large-scale setup with a human-sized anthropomorphic mobile robot.

Experiments in a 2 DoF Virtual Scenario with a linear haptic device, see Fig. 6, have been conducted as a user study yielding the results depicted in Fig. 4. The participants had to move a virtual point mass object of $100 \mathrm{~kg}$ through a simple maze from a starting configuration to a goal configuration through the scene without colliding with the virtual obstacles visually and haptically displayed. For quick haptic rendering of arbitrary scenes the scene information is imported from any standard monochrome pixel or vector graphics file and is automatically rendered as stiff environment. The control algorithm is implemented in Matlab/Simulink's Real-Time Workshop and executed on Linux Preempt/RT at a frequency of $1 \mathrm{kHz}$. Four conditions are tested: a) assisted by feedback planning, b) assisted by motion reproduction, and c) assisted by a combined strategy where motion reproduction is pre-initialized with simulated feedback planning, d) homotopy switching based on prediction certainty, and e) a multi-criterion decision making algorithm.

The virtual-reality interface consists of a two degrees-of-freedom (anteroposterior and mediolateral plane of the user standing in front) linear-actuated device (ThrustTube) with a freespinning handle (superoinferior direction of the user) at the grasp point. Attached to the handle, a force/torque sensor (JR3) measures the human contact force. The virtual scene is visually represented on a display placed on top of the interface, see Fig. 6. The scene information can be imported from virtually any standard monochrome pixel or vector graphics file and is automatically rendered as stiff environ-

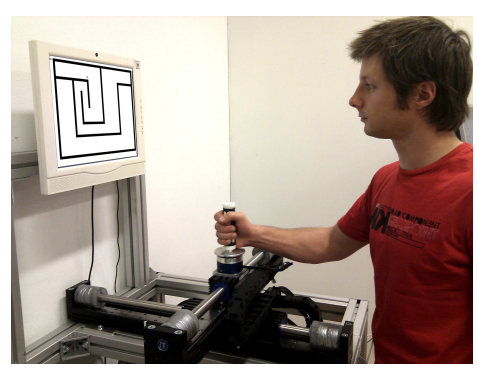

Fig. 6: 2-DoF VR platform ment. A virtual rigid polygon or ellipsoid object with distributed grasp points can be specified and simulated. 
Experiments in a full-scale scenario are conducted within the highly integrated CoTeSys multi-robot $l a b^{2}$ in exemplary domain of a carrepair and with the algorithms being transferred from the small scale setup. Practical challenges arise when the algorithms are applied to an actual cooperative load transport task. Properties such as scalability to higher degrees of freedom, robustness to sources of noise in autonomous mobile manipulation and safe behavior outside the expected motion corridor are evaluated in

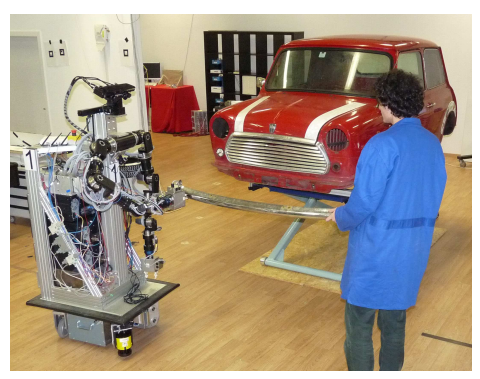

Fig. 7: Full-scale setup our highly integrated experimental system that has been instrumental to validate different aspects of human-robot collaboration. As an exemplary domain, we chose a classic-car repair setting. The mobile robot used in this experiment, see Fig. 7, locomotes with its four-wheeled omni-directional mobile platform which offers roughly human-like maneuverability and smooth motion [12]. Two identical anthropomorphic 7DoF manipulators provide a human-like working space [28]. Mounted onto a JR3 wrench sensor, the manipulator is equipped with a Schunk $P G 70$ two-finger parallel gripper which allows a tight grasp of the object. A point cloud of the environment is acquired using a tilted Hokuyo UBG laser range finder. Two Sick S300 laser range finders scan for obstacles above the surrounding floor. A ceiling camera system covering a space of $10 \times 10 \mathrm{~m}$ supports the localization of humans and robots. The cooperative transport of a Mini's steel bumper is considered through the cluttered lab environment, see Fig. 4 for a map. More information on the robot used can be found in [20]. The cooperatively manipulated object, a $1.2 \mathrm{~m}$ long Mini's steel bumper (b) weighing $1.9 \mathrm{~kg}$. The distance between the pre-defined grasp points of human and robot is $1.1 \mathrm{~m}$.

The software framework used in this experiment is based on the modular realtime architecture ARCADE [1] suited for rapid prototyping of perception-cognitionaction loops in complex human-robot teams scenarios. It interfaces to $R O S$ in a seamless manner; for example ROS algorithms are utilized for self-localization. The admittance-type control algorithm is implemented analogously to the VR setup and communicates with other software modules through the high-bandwidth real-time data base $R T D B$ [9] at a frequency of $1 \mathrm{kHz}$.

Similar to the VR scenario, a virtual admittance is rendered in world coordinates at the robot's end effector. The mobile platform locomotes such that the end effector can be controlled freely through the environment.

\footnotetext{
${ }^{2}$ See http: / /www. cotesys.org/newsroom/videos for videos of pHRI experiments.
} 


\section{Main Experimental Insights}

Our rapid prototyping environment is instrumental to identify a number of crucial properties relevant to the application of cooperative load transport and to identify strategies to exploit synergies between the two approaches investigated. Some of the complementary properties of the evaluated planning-based and learning-based approaches are presented in Table 2 with the focus on the motion generation.

Table 2: Qualitative properties of feedback motion planning (using SNG) and learning-based approach (using tHMM) for robotic partner behavior generation in physical human-robot cooperation

\begin{tabular}{|c|c|c|}
\hline Property & Planning & Learning-based approach \\
\hline Initial behavior & Goal oriented force ${ }^{a}$ & Observing \\
\hline Long-term behavior & Goal oriented force ${ }^{a}$ & $\begin{array}{l}\text { Precise reproduction of the hu- } \\
\text { man partner's preferred trajec- } \\
\text { tory, adapted to object dynamics }\end{array}$ \\
\hline $\begin{array}{l}\text { Behavior outside the pre- } \\
\text { ferred corridor }\end{array}$ & Goal oriented force ${ }^{a}$ & Observing \\
\hline Environment representation & Explicit for $\mathscr{C}$-space calculation & $\begin{array}{l}\text { Implicit/rough through training } \\
\text { data }\end{array}$ \\
\hline Parameter set & Desired velocity, precision & $\begin{array}{l}\text { Number of states; number of } \\
\text { Gaussians per state; weighting of } \\
\text { input importance }\end{array}$ \\
\hline Scalability & $\begin{array}{l}\text { Complexity of state space ap- } \\
\text { proximation grows exponentially } \\
\text { with output dimension }\end{array}$ & $\begin{array}{l}\text { Complexity grows linearly with } \\
\text { input dimension }\end{array}$ \\
\hline Computational effort & $\begin{array}{l}\text { Pre-computation effort per scene; } \\
\text { planning effort per new goal; neg- } \\
\text { ligible execution time effort }\end{array}$ & $\begin{array}{l}\text { Expectation maximization after } \\
\text { each observation; Viterbi and re- } \\
\text { gression during execution }\end{array}$ \\
\hline
\end{tabular}

${ }^{a}$ neglecting object dynamics and human preferences

Our large-scale experiment is used to validate the approaches presented in Section 3 in a lifelike scenario. ${ }^{3}$ For fast computation, parallelized implementations on an Intel Core i7 920 at $2.67 \mathrm{GHz}$ were utilized. The computation times for our prototypical 6D-problem are given in Table 3.

In conclusion we demonstrate that the proposed modular, layered framework for prototyping of pro-active physical robotic assistants embeds well various different approaches on different levels and gives an immediate chance to evaluate the interplay between modules. The evaluation in human user studies on a high-fidelity haptic interface allows for short development cycles and quick acquisition of scientific results. The transfer to the large-scale six-DoF scenario works seamlessly with computational complexity remaining the greatest challenge. In the future, we intend

${ }^{3}$ The video attachment shows the experimental setup used for validation of the planning-based and learning-based approaches. 
Table 3: Computation times of SNG and tHMM for robotic partner behavior generation in $6 \mathrm{D}$

\begin{tabular}{lcc}
\hline Computation step & SNG & tHMM \\
\hline Calculation of neighborhood graph from point cloud $\left(\alpha=P_{c}=0.985\right)$ & $639 \mathrm{~s}$ & \\
Planning per new goal configuration & $<1 \mathrm{~s}$ & \\
Expectation Maximization after each observation & $<5 \mu \mathrm{s}$ & $\sim 5 \mathrm{~s}$ \\
$\begin{array}{l}\text { Direction lookup per control iteration } \\
\text { Viterbi and regression during execution }\end{array}$ & $<500 \mu \mathrm{s}$ \\
\hline
\end{tabular}

to investigate more deeply possible options for the deliberative layer as it is to the best of the authors' knowledge a novel aspect in the context of physical human-robot interaction but shows great potential to enhance cooperation quality significantly.

Acknowledgements The authors would like to thank Dongheui Lee for supporting this work with valuable advice. This research is partly supported by the DFG excellence initiative research cluster "Cognition for Technical Systems - CoTeSys": www . cotesys . org.

\section{References}

1. D. Althoff, O. Kourakos, M. Lawitzky, A. Mörtl, M. Rambow, F. Rohrmüller, D. Brščić, D. Wollherr, S. Hirche, and M. Buss. An Architecture for Real-time Control in Multi-robot Systems. In Cognitive Systems Monog.: Human Centered Robot Systems, pages 43-52. 2009.

2. A. Billard, S. Calinon, R. Dillmann, and S. Schaal. Robot Programming by Demonstration. In B. Siciliano and O. Khatib, editors, Handbook of Robotics, pages 1371-1394. Springer, 2008.

3. S. Calinon, F. D'Halluin, E.L. Sauser, D.G. Caldwell, and A.G. Billard. Learning and reproduction of gestures by imitation. IEEE Robot. Autom. Mag., 17(2):44-54, 2010.

4. B. Corteville, E. Aertbelien, H. Bruyninckx, J. De Schutter, and H. Van Brussel. Humaninspired Robot Assistant for Fast Point-to-point Movements. In Proc. IEEE ICRA, pages 3639-3644, 2007.

5. C. Esteves, G. Archevaleta, and J. Laumond. Motion Planning for Human-Robot Interaction in Manipulation Tasks. In Proc. IEEE ICMA, pages 1766-1771, 2005.

6. P. Evrard and A. Kheddar. Homotopy Switching Model for Dyad Haptic Interaction in Physical Collaborative Tasks. In Proc. EHS EuroHaptics, pages 45-50, 2009.

7. V. Fernandez, C. Balaguer, D. Blanco, and M.A. Salichs. Active Human-Mobile Manipulator Cooperation Through Intention Recognition. In Proc. IEEE ICRA, pages 2668-2673, 2001.

8. T. Flash and N. Hogan. The coordination of arm movements: An experimentally confirmed mathematical model. J. Neurosci., 5:1688-1703, 1985.

9. M. Goebl and G. Färber. A Real-Time-capable Hard- and Software Architecture for Joint Image and Knowledge Processing in Cognitive Automobiles. In Proc. IEEE IV, pages 734 $740,2007$.

10. E. Gribovskaya, S.M. Khansari-Zadeh, and A. Billard. Learning Nonlinear Multivariate Dynamics of Motion in Robotic Manipulators. Int. J. Rob. Res., 30(1):80-117, 2010.

11. E. Gribovskaya, A. Kheddar, and A. Billard. Motion learning and adaptive impedance for robot control during physical interaction with humans. In Proc. IEEE ICRA, 2011.

12. U. Hanebeck, N. Saldic, and G. Schmidt. A Modular Wheel System for Mobile Robot Applications. In Proc. IEEE/RSJ IROS, pages 17-22, 1999. 
13. J.A. Ijspeert, J. Nakanishi, and S. Schaal. Movement Imitation with Nonlinear Dynamical Systems in Humanoid Robots. In Proc. IEEE ICRA, pages 1398-1403, 2002.

14. A. Kirsch, T. Kruse, E. Sisbot, R. Alami, M. Lawitzky, D. Brščić, S. Hirche, P. Basili, and S. Glasauer. Plan-Based Control of Joint Human-Robot Activities. Künstl. Intell., 24(3):223$231,2010$.

15. K. Kosuge and Y. Hirata. Human-Robot Interaction. In Proc. IEEE ROBIO, pages 8-11, 2004.

16. K. Kosuge, H. Yoshida, and T. Fukuda. Dynamic control for robot-human collaboration. In Proc. IEEE Workshop on Robot and Human Communication, pages 398-401, 1993.

17. M. Lawitzky, A. Mörtl, and S. Hirche. Load Sharing in Human-Robot Cooperative Manipulation. In Proc. IEEE Ro-Man, pages 185-191, 2010.

18. D. Lee and C. Ott. Incremental kinesthetic teaching of motion primitives using the motion refinement tube. Auton. Robot., pages 1-17, 2011.

19. Y. Maeda, T. Hara, and T. Arai. Human-robot cooperative manipulation with motion estimation. In Proc. IEEE/RSJ IROS, volume 4, pages 2240 -2245 vol.4, 2001.

20. J.R. Medina, M. Lawitzky, A. Mörtl, D. Lee, and S. Hirche. An Experience-Driven Robotic Assistant Acquiring Human Knowledge to Improve Haptic Cooperation. In Proc. IEEE/RSJ IROS, pages 2416-2422, 2011.

21. J.R. Medina, D. Lee, and S. Hirche. Risk Sensitive Optimal Feedback Control for Haptic Assistance. In Proc. IEEE ICRA, 2012 (accepted).

22. A. Mörtl, M. Lawitzky, A. Küçüky1lmaz, M. Sezgin, C. Basdogan, and S. Hirche. The Role of Roles: Physical Cooperation between Humans and Robots. Int. J. Robot. Res., 2012 (accepted).

23. P. Pastor, H. Hoffmann, T. Asfour, and S. Schaal. Learning and Generalization of Motor Skills by Learning from Demonstration. In Proc. IEEE ICRA, pages 1293-1298, 2009.

24. J. Rosell, C. Vázquez, A. Pérez, and P. Iñiguez. Motion Planning for Haptic Guidance. J. Intell. Robotic. Syst., 53(3):223-245, 2008.

25. T.L. Saaty. The Analytic Hierarchy Process. McGraw-Hill International, New York, NY, 1980.

26. S. Schaal and C. G. Atkeson. Constructive Incremental Learning From Only Local Information. Neural Computation, 10:2047-2084, 1997.

27. S. Schneider and R. Cannon. Object Impedance Control for Cooperative Manipulation: Theory and Experimental Results. IEEE Trans. Robot. Automat., 8(3):383-394, 1992.

28. B. Stanczyk and M. Buss. Development of a Telerobotic System for Exploration of Hazardous Environments. In Proc. IEEE/RSJ IROS, pages 2532-2537, 2004.

29. M. Taïx and D. Flavigné. Motion Planning with interactive devices. In Proc. IEEE ECMS, pages 1-6, 2011.

30. A. Thobbi, Y. Gu, and W. Sheng. Using Human Motion Estimation for Human-Robot Cooperative Manipulation. In Proc. IEEE/RSJ IROS, pages 2873-2878, 2011.

31. S. Vijayakumar, A. D'Souza, and S. Schaal. Incremental Online Learning in High Dimensions. Neural Computation, 17:2602-2634, 2005.

32. J.M. Wang, D.J. Fleet, and A. Hertzmann. Gaussian process dynamical models for human motion. IEEE Trans. Pattern Anal. Mach. Intell., 30(2):283-298, 2008.

33. L. Yang and S. LaValle. The Sampling-Based Neighborhood Graph: An Approach to Computing and Executing Feedback Motion Strategies. IEEE Trans. Robot. Automat., 20(3):419-432, 2004.

34. T. Yoshikawa and $\mathrm{O}$. Khatib. Compliant motion control for a humanoid robot in contact with the environment and humans. In Proc. IEEE/RSJ IROS, pages 211-218, 2008. 


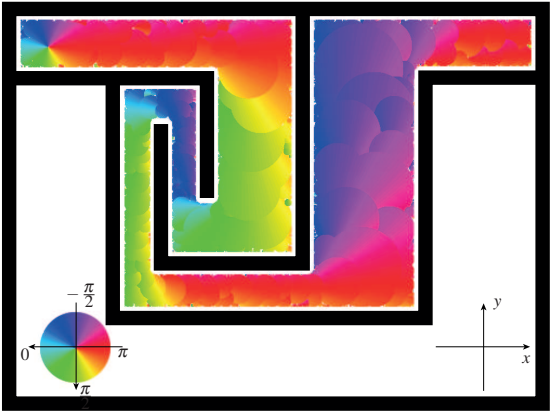

(a) Feedback motion plan

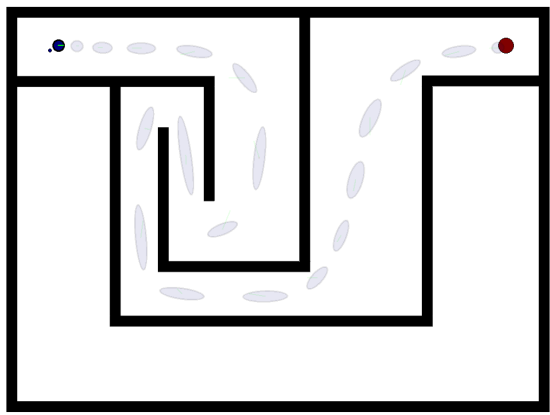

(c) Left-to-right HMM

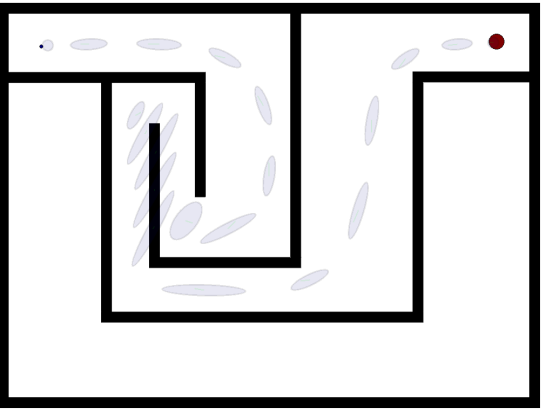

(b) Ergodic HMM

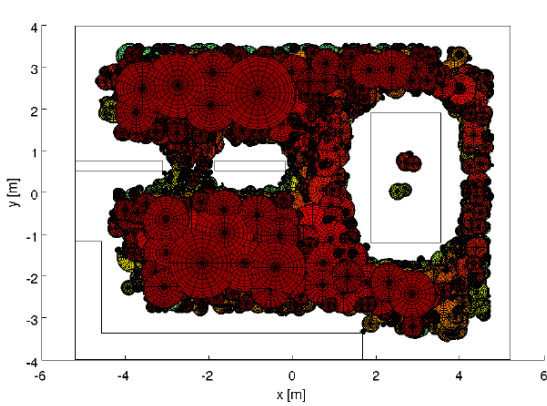

(d) $x / y / \phi$ components of feedback motion plan

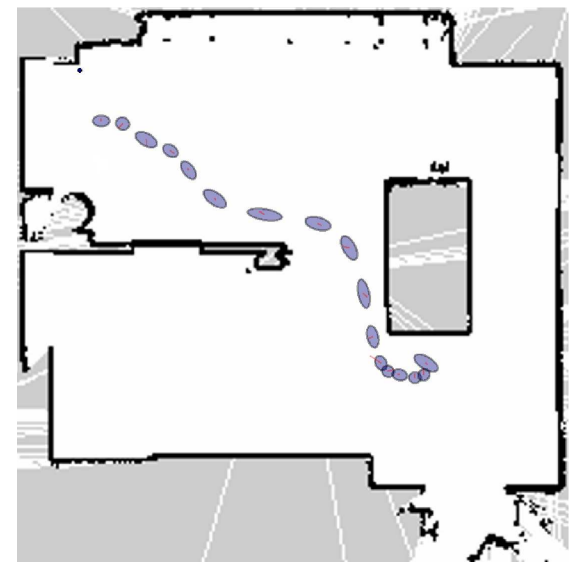

(e) $x / y$ components of distributed Gaussians

(f) Magnified central region of Fig. 5e

Fig. 5: Motion generation algorithms in a 2DoF virtual haptic maze (a)-(c), and in $6 \mathrm{DoF}$ in the $10 \times 10 \mathrm{~m}$ cluttered labspace mapped by a laser-range finder (d)-(f). 